\title{
Minimally invasive ascending aorta replacement
}

\author{
Öztekin Oto ${ }^{1}$, Kıvanç Metin ${ }^{2}$
}

\footnotetext{
1) Dokuz Eylul University Faculty of Medicine, Department of Thoracic and Cardiovascular Surgery / Izmir / Turkey

2) Ege Sağlık Hospital Department of Cardiovascular Surgery, İzmir, Turkey
}

\section{Summary}

56-year-old female patient weighing $80 \mathrm{~kg}$ was hospitalized to have an operation for aneurysmal expansion in ascending aorta (47 mm in TTE) detected in the examinations performed for complaints of chest and back pain. No other cardiological pathology was detected. Minimally invasive technique was preferred for the replacement of ascending aorta. Differently from aortic valve replacement, peripheral cannulation was preferred for arterial cannulation. Right brachial artery was explored with a $2-\mathrm{cm}$ incision in axillary region from distal section of axillary artery and cannulated with $18 \mathrm{~F}$ peripheral artery cannula. A "two stage" venous cannula was inserted through right atrial appendix. During cardiopulmonary bypass for venous return, vacuum between 20 and $40 \mathrm{mg} \mathrm{Hg}$ was applied. Left atrial vent cannulas were also inserted via cardioplegia and left upper pulmonary vein. During the procedure, appropriate pump output (2.2-2.4 $\mathrm{lt} / \mathrm{min} / \mathrm{m} 2)$ was established for the patient without any complication. Ascending aorta was replaced with vasculary graft of $24-\mathrm{mm}$ diameter. Left ventricle was transeptally deaired through right ventricle in deep Trendelenburg position and external cardioversion was applied. After cardiopulmonary bypass was finished, brachial artery was primarily repaired with $5 / 0$ polypropylene during decannulation.

Keywords: Minimally invasive, aorta replacement, cardiopulmonary bypass

\section{Case}

56-year-old female patient weighing $80 \mathrm{~kg}$ was hospitalized to have an operation for aneurysmal expansion in ascending aorta (47 $\mathrm{mm}$ in TTE) detected in the examinations performed for complaints of chest and back pain. No other cardiological pathology was detected. Minimally invasive technique was preferred for the replacement of ascending aorta. Differently from aortic valve replacement, peripheral cannulation was preferred for arterial cannulation. Right brachial artery was explored with a $2-\mathrm{cm}$ incision in axillary region from distal section of axillary artery and cannulated with $18 \mathrm{~F}$ peripheral artery cannula. A "two 
stage" venous cannula was inserted through right atrial appendix. During cardiopulmonary bypass for venous return, vacuum between 20 and $40 \mathrm{mg} \mathrm{Hg}$ was applied. Left atrial vent cannulas were also inserted via cardioplegia and left upper pulmonary vein. During the procedure, appropriate pump output (2.2-2.4 $1 \mathrm{t} / \mathrm{min} / \mathrm{m} 2)$ was established for the patient without any complication. Ascending aorta was replaced with vasculary graft of 24-mm diameter. Left ventricle was transeptally deaired through right ventricle in deep Trendelenburg position and external cardioversion was applied. After cardiopulmonary bypass was finished, brachial artery was primarily repaired with $5 / 0$ polypropylene during decannulation.

The patient was taken into intensive care unit without any complication, then taken into normal clinical care on postoperative day one, and discharged in day five. There is no problem in the follow-ups as of postoperative week four.

\section{Discussion}

Aortic valve surgical interventions can be performed with minimally invasive techniques using partial sternotomies, parasternal incisions, mini-thoracotomies and transverese sternotomy incisions. Among these techniques, mini-sternotomy incisions which provide standard surgical appearance, stand out. Semi-sternotomy incisions limited to upper and lower ends, may be applied, and topographically, sufficient surgical opinion is obtained on the cardiac region to be intervened.

We are routinely applying aortic valve surgical interventions with sternotomy incision limited to upper end. Upper end mini sternotomy incision is appropriate for standard surgical equipment, and does not require additional technique investment. As sternum stability is protected, the risk for bone-related complications in postoperative period is less and patients have shorter recovery periods without any complications. Internal throcic artery injury is also a rare condition. Sufficient exposure can be obtanied for aorta surgery with short sternotomies less than $5 \mathrm{~cm}$ involving manubrium sterni.
Still, due to necessary cannulation procedure for installing cardiovasculary bypass connection, working area is reduced. When we started to do minimally invasive surgical intervention, we were preferring central cannulation for cardiopulmonary bypass. As cannulas narrow down the surgical area, search for alternative techniques became inevitable. Performing the surgical interventions with small incision without compromising the standard surgical technique is possible with some variations such as insertion of venous cannula through the incision used for mediastinum drain.

A major limitation related with limited sternotomy is the difficulties in positioning the heart. After removal of cross clamp, no manipulation can be performed for deairing of left ventricle apex or for cardioversion. For these, there are various alternative solutions: Transseptal deairing through right ventricle or external cardioversion with two cautery plates attached to the patient's back (in a direction consistent with cardiac axis) in preparation period before the surgery.

With the established solutions for the above mentioned limitations, the many benefits of minimally invasive surgery were understood better. Limited incision provides a fast healing period without complications especially in elderly patients as it protects sternal stability. It causes less pain, patient can be mobilized earlier and become self-sufficient. Smaller incision causes less bleeding and thereby, provides medical and economical advantages such as reduced transfusion requirement or shortened period for staying in intensive care unit or hospitalization.

By using alternative arterial cannulation and single venous cannulation for ascending aorta replacement, we removed the cannulas restricting the surgical area out of the site and successfully used the upper end mini-sternotomy incision. The innovative and "inventive" nature of our occupation makes being creative inevitable. Finding ways for applying successful and safe surgical intervention methods by using less invasive techniques and applying them with courage establish the future of minimally invasive cardiac surgery.

Corresponding author: Dr. Öztekin Oto

Mail: oztekinoto@oztekinoto.com

\section{Disclosure and conflicts of interest: \\ Conflicts of interest were not reported.}

